\title{
DIHEDRAL SINGULARITIES: INVARIANTS, EQUATIONS AND INFINITESIMAL DEFORMATIONS
}

BY OSWALD RIEMENSCHNEIDER

Communicated by Robert Fossum, May 7, 1976

In this note we give a short survey on joint work with $\mathrm{K}$. Behnke; details will appear in [1] and [2].

Let $n, q$ be positive integers with $2 \leqslant q<n$ and $\operatorname{gcd}(n, q)=1, m=n-q$. We define elements $\phi_{m}, \psi_{q}, \eta \in \mathrm{GL}(2, \mathrm{C})$ by

$$
\phi_{m}=\left(\begin{array}{cc}
\zeta_{2 m} & 0 \\
0 & \zeta_{2 m}
\end{array}\right), \quad \psi_{q}=\left(\begin{array}{cc}
\zeta_{2 q} & 0 \\
0 & \zeta_{2 q}^{-1}
\end{array}\right), \quad \eta=\left(\begin{array}{cc}
0 & i \\
i & 0
\end{array}\right)
$$

where $i=\sqrt{-1}$ and $\zeta_{k}=\exp (2 \pi i / k)$. The group $G_{n, q} \subset \operatorname{GL}(2, \mathbf{C})$ is generated by

(a) $\phi_{m}, \psi_{q}, \eta$ in case $m$ odd,

(b) $\psi_{q}, \eta \circ \phi_{2 m}$ in case $m$ even.

$G_{n, q}$ has finite order $4 m q ; G_{q+1, q}$ is the binary dihedral group of order $4 q$. $G_{n, q}$ acts on $\mathbf{C}^{2}$ in the usual way; the quotient $\mathbf{C}^{2} / G_{n, q}$ has precisely one (normal) complex-analytic singularity. We call it the dihedral singularity of type $D_{n, q}$. If we expand $n / q$ into the modified continued fraction à la HirzebruchJung,

$$
n / q=b_{3}-1 \sqrt{b_{4}}-\cdots-1 \sqrt{b_{r}}, \quad b_{\rho} \geqslant 2, r \geqslant 4
$$

it can be characterized by the dual graph of its minimal resolution (cf. [3]):

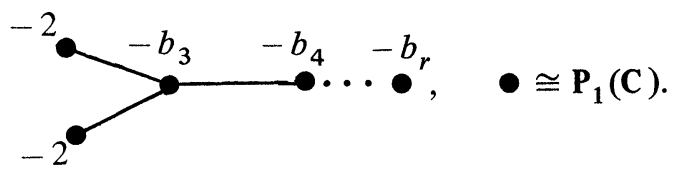

The equations are calculated by invariant theory. As in the cyclic group case [5], we put

$$
n / m=a_{2}-1 \sqrt{a_{3}}-\cdots-1 \sqrt{a_{e-1}}, \quad a_{\epsilon} \geqslant 2 .
$$

Further set $A_{3}=a_{3}+1, A_{\epsilon}=a_{\epsilon}, \epsilon \neq 3$, and

AMS (MOS) subject classifications (1970). Primary 32C40, 32G05, 14B05, 14J15; Secondary $32 \mathrm{C} 45,14 \mathrm{E} 15$. 


$$
\begin{array}{cc}
s_{2}=1, s_{3}=1, \quad s_{\epsilon+1}=A_{\epsilon} s_{\epsilon}-s_{\epsilon-1}, & 3 \leqslant \epsilon \leqslant e-1, \\
t_{2}=a_{2}, t_{3}=a_{2}-1, t_{\epsilon+1}=A_{\epsilon} t_{\epsilon}-t_{\epsilon-1}, & 3 \leqslant \epsilon \leqslant e-1, \\
r_{\epsilon}=m t_{\epsilon}-q s_{\epsilon}, & 2 \leqslant \epsilon \leqslant e-1 .
\end{array}
$$

Then we have

THEOREM 1. A minimal set of generators for $S_{n, q}=\mathbf{C}[u, v]^{G} n, q$ is formed by the polynomials

$$
z_{1}=(u v)^{2 m}, \quad z_{\epsilon}=(u v)^{r} \epsilon\left(u^{2 q s_{\epsilon}}+(-1)^{t} \epsilon^{2 q s} \epsilon\right), \quad \epsilon=2, \ldots, e .
$$

After a (noncanonical) change of variables it is possible to find simple equations.

THEOREм 2. The dihedral singularity of type $D_{n, q}$ is (minimally) described by $1 / 2(e-1)(e-2)$ equations

$$
\begin{aligned}
& z_{2}^{2}=z_{1}\left(z_{3}^{2}+z_{1}^{a_{2}-1}\right) \\
& z_{1} z_{\epsilon}=z_{2} z_{3}^{a_{3}-2} \cdots z_{\epsilon-2}^{a}{ }^{-2} z_{\epsilon-1}^{a}{ }^{a}, \quad \quad \epsilon=4, \ldots, e, \\
& z_{2} z_{\epsilon}=z_{3}^{a_{3}-2} \cdots z_{\epsilon-2}^{a}{ }^{-2} z_{\epsilon-1}^{a_{\epsilon-1}-1}\left(z_{3}^{2}+z_{1}^{a_{2}-1}\right), \quad \epsilon=4, \ldots, e, \\
& z_{\epsilon-1} z_{\epsilon+1}=z_{\epsilon}^{a} \epsilon, \quad \epsilon=4, \ldots, e-1, \\
& z_{\delta} z_{\epsilon}=z_{\delta+1}^{a_{\delta+1}-1} z_{\delta+2}^{a_{\delta+2}-2} \cdots z_{\epsilon-2}^{a}{ }^{-2} z_{\epsilon-1}^{a_{\epsilon-1}-1}, \\
& 4 \leqslant \delta+1<\epsilon-1 \leqslant e-1 .
\end{aligned}
$$

In the case $e=4$ these equations are given by the maximal subdeterminants of the $3 \times 2$-matrix

$$
\left(\begin{array}{ccc}
z_{1} & z_{2} & z_{3}^{a_{3}-1} \\
z_{2} & z_{3}^{2}+z_{1}^{a_{2}-1} & z_{4}
\end{array}\right) .
$$

This is in accordance with (and proved by) Wahl's theorem on equations defining rational singularities [6].

For the computation of $T^{1}$, the vector space of infinitesimal deformations, we use Pinkham's method [4]. In [1] we reduce the problem to the solution of a (large) system of linear equations and give some examples. A general formula for the dimension of $T^{1}$ will be proved in [2]:

THEOREM 3.

$$
\operatorname{dim} T^{1}=\sum_{\epsilon=2}^{e-1} a_{\epsilon}+c
$$


where

$$
c= \begin{cases}1, & e=3, \\ 2, & a_{3}=2, \\ 3, & a_{3} \geqslant 3 .\end{cases}
$$

In another forthcoming manuscript we determine the invariants and equations for all remaining quotient surface singularities.

\section{REFERENCES}

1. K. Behnke and O. Riemenschneider, Diedersingularitäten, Abh. Math. Sem. Univ. Hamburg (to appear).

2. - Infinitesimale Deformationen von Diedersingularitäten (in preparation).

3. E. Brieskorn, Rationale Singularitäten komplexer Flächen, Invent. Math. 4 (1967/68), 336-358. MR 36 \#136.

4. H. Pinkham, Deformations of quotient surface singularities, Proc. Sympos. Pure Math., vol. 30, Part 1 (Proc. Conf. on Several Complex Variables, Williamstown, 1975), Amer. Math. Soc., Providence, R. I. (to appear).

5. O. Riemenschneider, Deformationen von Quotientensingularitäten (nach zyklischen Gruppen), Math. Ann. 209 (1974), 211-248. MR 51 \#3518.

6. J. Wah1, Equations defining rational singularities, 1975 (preprint).

MATHEMATISCHES SEMINAR DER UNIVERSITÄT HAMBURG, BUNDESSTRASSE 55, 2000 HAMBURG 13, WEST GERMANY 